\title{
User evaluation and eye tracking-based prediction model for tractor hood product design
}

\author{
Yujiao $\mathrm{Wu}^{1}$, Feng Zheng ${ }^{1 *}$ \\ ${ }^{1}$ School of mechanical and automotive engineering, Qilu University of Technology, Jinan, Shandong, 250353, China
}

\begin{abstract}
To objectively evaluate the product design of tractor hoods, they have been set as variables, and the remaining components of the hood have been taken as the rations. Eye-tracking and semantic differencebased experiments were performed to determine the level of attention a user gave to the hood and an image evaluation value for the same; morphological analysis was used to deconstruct the structural elements of the tractor hood. The structural elements and image evaluation values were implemented as input and output layers, respectively, in a back-propagation neural network (BPNN) used to train and verify a user-evaluation prediction model for tractor hood designs. The results show that the BPNN model can accurately predict a user's evaluation of the tractor hood design, thereby providing a reference for designers in terms of the tractor hood shape, and quantify user evaluations of the hood design.
\end{abstract}

\section{Introduction}

For a long time, manufacturers of agricultural machinery have paid attention to product functionality at the expense of structural design. In terms of power equipment commonly used in agricultural production, the wheeled tractor is particularly prominent [1]. The scope of China's modernization of agricultural equipment has rapidly expanded recently and the demand for tractors has continued to grow. Chinese and foreign tractor enterprises face increasingly fierce competition with the expansion of the global sales market. To distinguish themselves, it is necessary to improve form design quality, enhance the aesthetics of the machine to better appeal to possible consumers, and meet the needs of users [1-2]. A tractor hood, as the main covering component of the tractor, houses the engine and other components and provides safe working conditions for the operator, which is the primary consideration in tractor design [3]. Therefore, hood design is the key in the process of creating a new tractor.

Eye movement is considered to be the most effective method in the field of visual information processing research [4-6]. In recent years, eye-tracking research has been widely used in product design research $[7,8]$. Li et al. [9] developed a visual cognition law for humanoid robot imagery by performing an eye-tracking experiment and used a subjective sentiment measurement evaluation method to explore the intrinsic relationship between humanoid robot model imagery and user cognition. Lu et al. [10] used an eye-tracking method to select important eye movement indicators and explored the influence of different completed design works and the professional background of students on eye movement behavior for application to design decisions. Su et al. [11] established an evaluation model for product design elements using eye-movement data as a physiological indicator of consumer interest; they then compared the consumer psychological experience results to rank the contribution of the various design elements of a machine. Zhou [12] used an eye tracker to perform two-eye tests without a target or task; they obtained the key features of an industrial robot that significantly influenced the cognitive process of the subject and determined the significance of the structural elements of the industrial robot based on the target intention.

Back-propagation neural networks (BPNNs) implement a back-propagation algorithm to reduce error. The network possess an input layer, a hidden layer, and an output layer. As shown in Figure 1, it has strong nonlinear mapping ability, and is commonly used to establish the complex relationship between input variables and output variables [13]. Based on relevant research so far, BPNNs have been applied to design product elements and evaluate the perception of product design imagery. Zhu et al. [13] analyzed the relationship between the design elements comprising the head shape of a service robot, and the perception-based image evaluation of the subject; they then fitted the relationship model, implemented it in 3D modeling software, and developed an auxiliary product design system for the head shape design of the service robot. Li et al. [14] used a 20 -in. folding bicycle as an example to obtain product design elements and perception-based image evaluation values using a structural analysis method and a Likert scale questionnaire; the results were used to construct a BPNN to model the relationship between product design and perceived imagery. Su et al. [15] carried out an example study on the design of a goblet, established a 
BPNN to model the relationship between the design parameters and perceived image of the goblet, and embedded the model into a Computer Aided Design (CAD) system to construct a product design system that provides timely feedback about what part of the image is being perceived by the subject. Chen [16] used a dual BPNN to model the relationship between the appearance of the elements and appearance of the car; they then established a neural network model to relate appearance and consumer satisfaction, and finally associated the two neural network models to create an image perceptionbased prediction model of the product modeling design scheme.

At present, there are only a few reports on research related to the perceptual evaluation of tractor hood design, and there is also a lack of quantitative prediction methods for the evaluation of tractor hood designs. Therefore, in this study, a BPNN was applied to the modeling and prediction tractor hood designs. The results of an eye-tracking experiment were able to prove that the tractor hood component is the visual focus of a person observing a tractor. An oral evaluation was conducted to obtain a subject's perceived evaluation value for the tractor hood shape. The individual elements that make up a tractor hood were deconstructed by applying the form analysis method to establish a model that could quantify the relationship between the tractor hood design and the subject's perception-based evaluation; this provides a reference for tractor hood design.

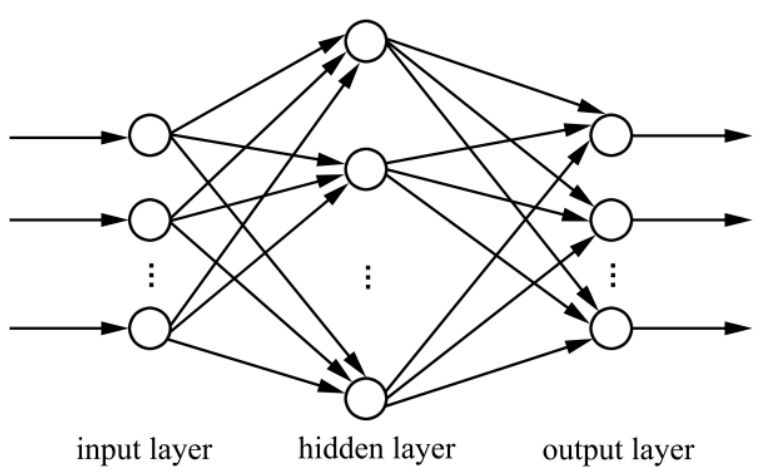

Figure 1. BPNN model.

\section{Research material preparation}

\subsection{Sample collection and sorting}

We first collected tractors from manufacturers such as John Deere, DEUTZ Farr, New Holland, Kubota, Lovol, and Dongfanghong through various channels. For consistency, we used only wheeled tractors as the research object and collected 155 samples of these with $45^{\circ}$ viewing angles. In terms of image selection, we did not include pictures with a disordered background, similar samples, or unclear samples. Next, we established a focus group consisting of 10 industrial design postgraduates and undergraduates with professional training and product design experience, according to modeling induction to reduce the number of samples to 100. The affinity graph method (i.e., the KJ method) was used to study 40 industrial design postgraduates and undergraduates. After eliminating invalid response, multi-scale and cluster analyses were performed on 38 groups of valid data; 10 representative wheeled tractors were consequently obtained, as shown in Figure 2. As the samples were implemented in eye-tracking tests in subsequent studies, they were gray-scaled to eliminate the influence of color on eye movement [10].

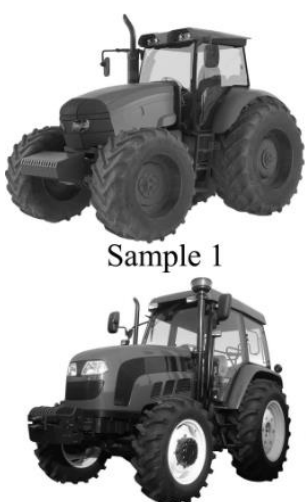

Sample 6

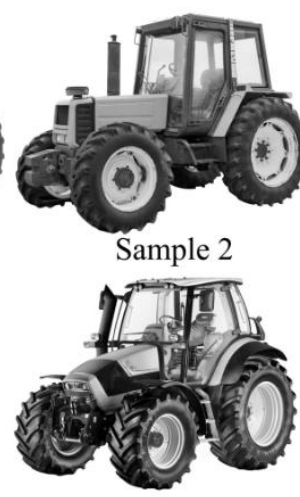

Sample 7

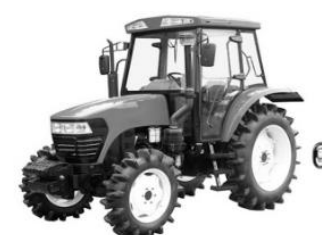

Sample 3

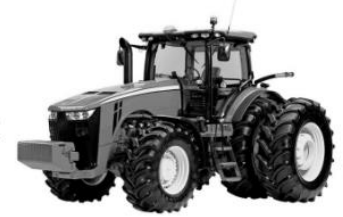

Sample 8

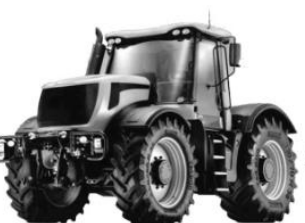

Sample 4

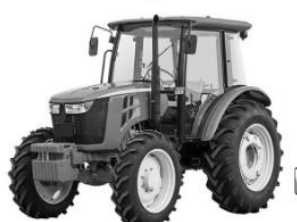

Sample 9
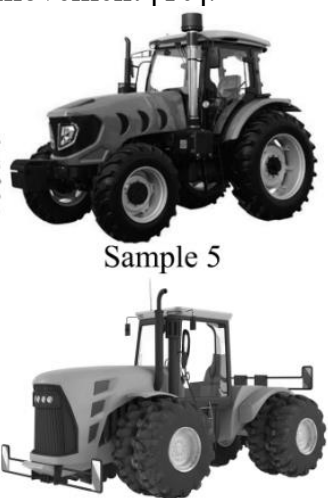

Sample 10

Figure 2. Representative sample images. 


\subsection{Collection and screening of perceptual images}

For evaluation, 124 perception-related words on tractor design were collected and processed by applying

Table 1. Total variance interpretation of factor analysis. antisense pairing. Following an online survey questionnaire, 14 groups (28 words) were initially selected and 10 tractor samples were selected for application in a semantic survey questionnaire [17]. The results of factor analysis are shown in Tables 1 and 2.

\begin{tabular}{|c|c|c|c|c|c|c|c|c|c|}
\hline \multirow{2}{*}{ element } & \multicolumn{3}{|c|}{ Starting eigenvalue } & \multicolumn{3}{|c|}{ Extract square sum load } & \multicolumn{3}{|c|}{ Cyclic sum of squares loading } \\
\hline & Total & variation $\%$ & accumulation $\%$ & Total & variation $\%$ & accumulation $\%$ & Total & variation $\%$ & accumulation $\%$ \\
\hline 1 & 6.871 & 49.077 & 49.077 & 6.871 & 49.077 & 49.077 & 4.474 & 31.958 & 31.958 \\
\hline 2 & 3.243 & 23.166 & 72.242 & 3.243 & 23.166 & 72.242 & 4.240 & 30.287 & 62.244 \\
\hline 3 & 1.897 & 13.552 & 85.794 & 1.897 & 13.552 & 85.794 & 3.297 & 23.550 & 85.794 \\
\hline 4 & 0.726 & 5.185 & 90.979 & & & & & & \\
\hline 5 & 0.543 & 3.880 & 94.860 & & & & & & \\
\hline 6 & 0.356 & 2.543 & 97.403 & & & & & & \\
\hline 7 & 0.200 & 1.427 & 98.830 & & & & & & \\
\hline 8 & 0.121 & .862 & 99.691 & & & & & & \\
\hline 9 & 0.043 & .309 & 100.000 & & & & & & \\
\hline 10 & $2.170 \mathrm{E}-16$ & $1.550 \mathrm{E}-15$ & 100.000 & & & & & & \\
\hline 11 & $1.885 \mathrm{E}-16$ & $1.347 \mathrm{E}-15$ & 100.000 & & & & & & \\
\hline 12 & $-4.884 \mathrm{E}-17$ & $-3.489 \mathrm{E}-16$ & 100.000 & & & & & & \\
\hline 13 & $-1.755 \mathrm{E}-16$ & $-1.254 \mathrm{E}-15$ & 100.000 & & & & & & \\
\hline 14 & $-4.047 \mathrm{E}-16$ & $-2.891 \mathrm{E}-15$ & 100.000 & & & & & & \\
\hline
\end{tabular}

Table 1 shows that the cumulative contribution of the first 3 factors was $85.794 \%$, indicating that they contained most of the essential information comprising the 14 groups of perception-related words; additionally, the variance contribution of each of these factors was found to be $49.077 \%, 23.166 \%$, and $13.552 \%$, respectively. This 4:2:1 proportion was used as a reference in the final selection of perception-related words.

Table 2. Post-rotation factor load matrix.

\begin{tabular}{cccc}
\hline & \multicolumn{3}{c}{ Element } \\
\cline { 2 - 4 } streamlined-geometric & 1 & 2 & 3 \\
cheap-expensive & 0.879 & 0.295 & 0.187 \\
low-end-high-end & 0.854 & -0.374 & -0.206 \\
regressive-advanced & 0.831 & -0.434 & -0.071 \\
domestic-international & 0.811 & -0.488 & -0.093 \\
solemn-dynamic & 0.733 & -0.175 & 0.534 \\
clumsy-light & -0.106 & 0.934 & -0.002 \\
outdated-innovative & 0.040 & 0.840 & -0.302 \\
exaggerated-simple & 0.321 & -0.756 & -0.409 \\
progressive-conservative & -0.545 & 0.742 & 0.313 \\
traditional-modern & -0.545 & 0.661 & 0.300 \\
thin-sturdy & 0.464 & -0.608 & -0.413 \\
perishable-durable & -0.049 & 0.071 & 0.953 \\
tough-flexible & -0.199 & -0.020 & 0.869 \\
\hline
\end{tabular}




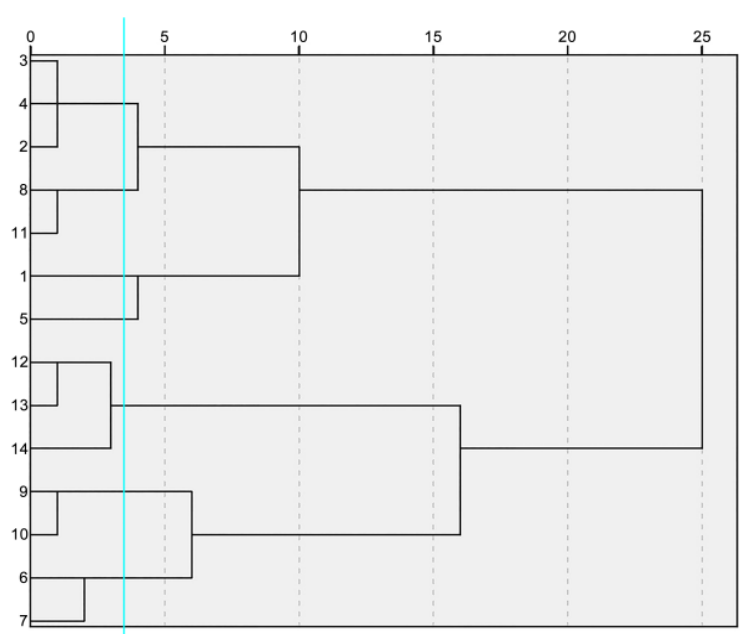

Figure 3. Cluster analysis tree for images.

The selection of the final representative words was performed considering the results presented in Table 2 and Figure 3. According to the factor load matrix size (Table 2), and the grouping bounds marked by the straight line in Figure 2, seven representative word groups, i.e., "solemn-dynamic," "exaggerated-simple," "thin-sturdy," "streamlined - geometric," "traditional_ modern," "low-end-high-end," and "domesticinternational," were selected for subsequent evaluation of the imagery.

\section{Experimental design}

Two types of eye-tracking experiments were conducted: a free eye-movement experiment (Experiment 1) and task-based eye-movement experiment (Experiment 2). The first type entailed unrestricted observation of the tractor sample images; the aim was to verify the cognitive process associated with visually focusing on the position of the tractor hood. Based on the results of the free eye-movement experiment, the hood component was considered to be the main variable; the remaining tractor components were quantified to properly process the sample image of each tractor. The second type of experiment was performed for a comparative analysis of the visual focus of the processed sample image of each tractor. Additionally, the purpose of the oral assessment was to obtain the perception-based evaluation value for tractor hood design from each subject; these were then used to study the relationship between the tractor hood design and subject perception of the image.

\subsection{Experimental object}

The group of students (i.e., subjects) recruited to participate in the eye-movement experiment was composed of 40 industrial design postgraduates and undergraduates. The age range was 20-25 yrs. Their vision can meet the experiment requirements after correction. The subjects were all right-handed.

\subsection{Experimental equipment}

In the experiment, Tobii desktop eye-tracking technology was used to record subjects' eye movement data as they observed different sample images; the experimental steps and data analysis for the eye movement experiment were performed using Eyeso Studio 3.0 software.

\subsection{Experimental design of free eye-movement experiment}

3.3.1 Experimental design and process. The 10 tractor sample images were displayed individually and the subject was free to observe the sample image. They were instructed to press the left mouse button to switch to the next image. The aim was to determine whether the subject would focus on the tractor hood when observing the tractor.

3.3.2 Analysis of experimental results. Figure 4 shows the heat map that was calculated using the eyemovement data from all the subjects. It shows that when the subjects freely observed the sample image of each tractor, its hood was the focus of their attention.

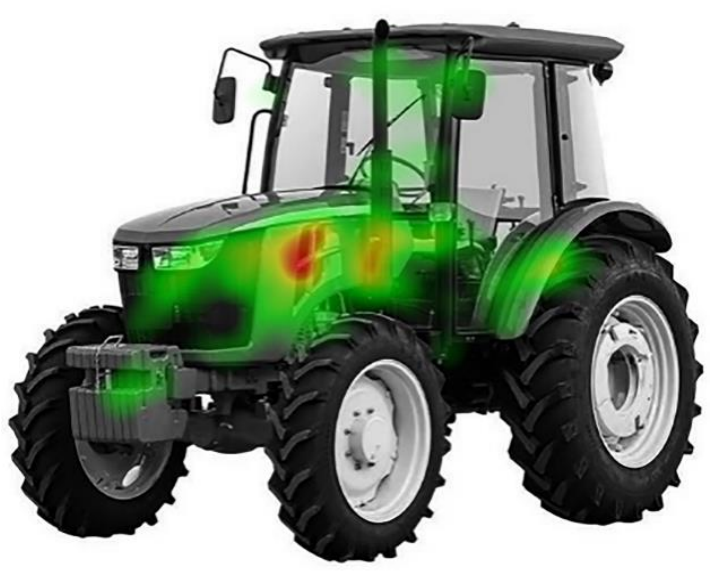

Figure 4. Heat map for a sample image.

\subsection{Task-based eye-movement experimental design}

As previously mentioned, the results of Experiment 1 show that when subjects observed the sample images, the tractor hood was the design feature they focused on most. Therefore, in Experiment 2, the tractor hoods were set as variables and the remaining tractor components were quantified to allow the study of the relationship between the tractor hood design and how each subject perceived the image. According to the results of the previously mentioned semantic survey questionnaire, Tractor No. 1 received a value that was close to the median and its design had no distinct features. Therefore, Tractor No. 1 was selected as the reference sample; it was grayscaled to remove any remaining distinguishing features. The hood of silhouetted Tractor No. 1 was then transformed into the hood of other 9 tractor samples sequentially to form a new experimental image for each sample (Figure 
5). The 10 new experimental sample images were then displayed on the same screen as the non-normalized images to generate different perception-based data for comparative analysis. The subjects also provided an oral evaluation of each sample image as they were instructed to answer relevant questions about each image for a subjective analysis. The same subjects and experimental instruments used in Experiment 1 were selected for the task-based eye-movement experiments. The semantic evaluation results obtained via the experiment were averaged and processed as shown in Table 3. The eyemovement data for all subjects were then processed to obtain a sample heat spot map for each sample tractor image, as shown in Figure 6.

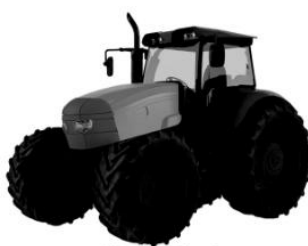

Sample 1

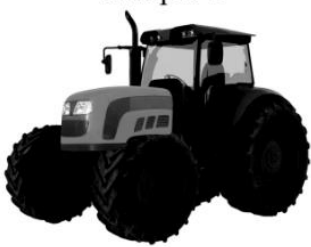

Sample 6

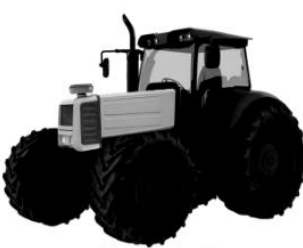

Sample 2

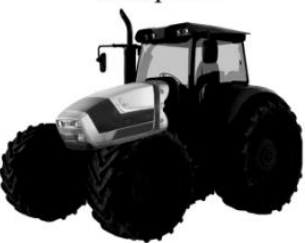

Sample 7

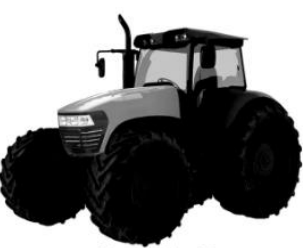

Sample 3

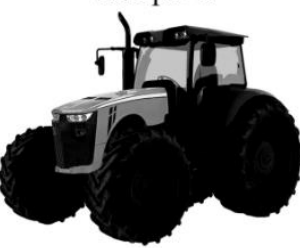

Sample 8

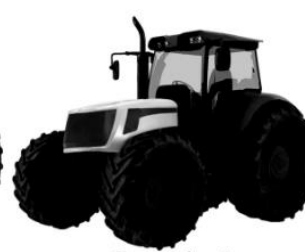

Sample 4

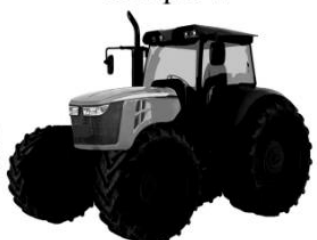

Sample 9

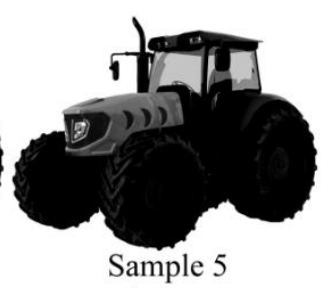

Sample 5

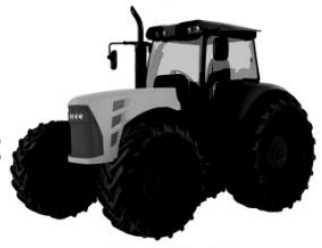

Sample 10

Figure 5. Sample images used in the task-based experiment.

Table 3. Semantic evaluation results.

\begin{tabular}{cccccccc}
\hline & Sample 1 & Sample 2 & Sample 3 & $\ldots$ & Sample 8 & Sample 9 & Sample 10 \\
\hline solemn-dynamic & 0.75 & -2.75 & 1.5 & $\ldots$ & 0.75 & -1 & -1 \\
exaggerated-simple & 0.75 & 2.25 & 1 & $\ldots$ & -1.5 & 0.75 & 0 \\
thin-sturdy & 0.75 & 1.25 & -0.25 & & 0.75 & 0.25 & 0.5 \\
streamlined-geometric & -1.5 & 3 & -1.5 & & -1 & -1.25 & 0.25 \\
traditional-modern & -0.25 & -1.5 & -0.5 & & 0.25 & 0.25 & 0.5 \\
low-end-high-end & 1.25 & -0.5 & 0.25 & $\ldots$ & 1 & 0 & 0.5 \\
domestic-international & 0.75 & -0.5 & -0.5 & $\ldots$ & 0.25 & -0.75 & 0.5 \\
\hline
\end{tabular}

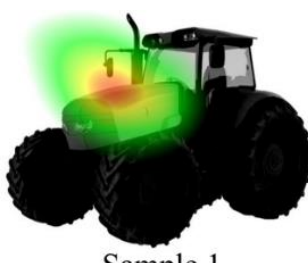

Sample 1

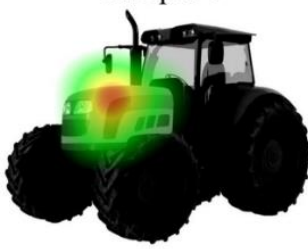

Sample 6

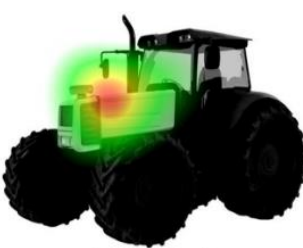

Sample 2

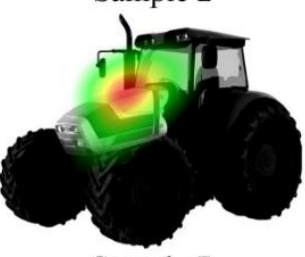

Sample 7

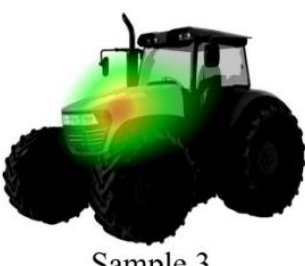

Sample 3

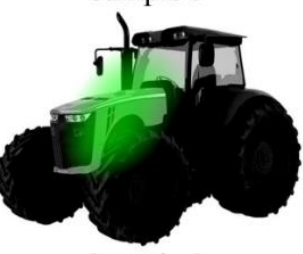

Sample 8

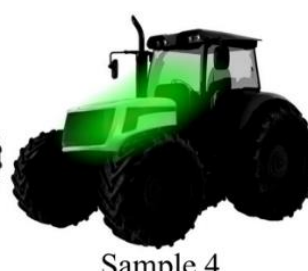

Sample 4

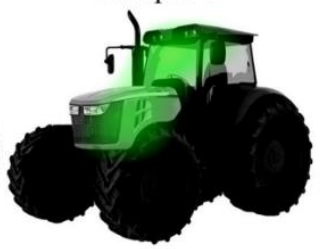

Sample 9

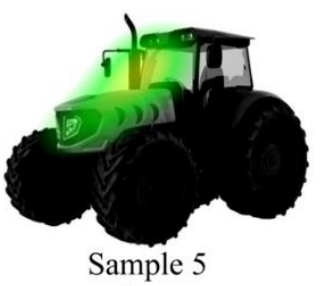

Sample 5

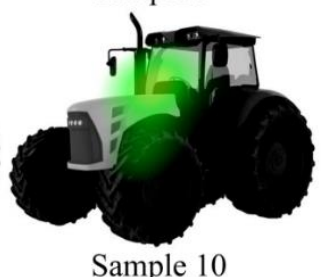

Sample 10

Figure 6. Heat map results for task-based experimental images.

It can be ascertained from the heat maps shown in Figure 6 that the results of the observation and semantic evaluation revealed that the subjects considered the hood to be the most important feature of each sample tractor image; therefore, the evaluation value can be considered to be the result of perception-based image evaluation of the hood. Each subject's fixation duration data for each sample image were imported into SPSS software for one- way analysis of variance (ANOVA). The results of the analysis are presented in Tables 4 and 5, and Figure 7; as given, $\mathrm{F}=2.333$, and $\mathrm{p}=0.016<0.05$, indicating that there was a significant difference in the duration of visual fixation on the tractor hood when the subjects were observing and evaluating perception-related words with "solemn—dynamic" as the target. 
Table 4. Test results for homogeneity of variance.

\begin{tabular}{ccccc}
\hline & Levene Statistic & df1 & df2 & Significance \\
\hline Fixation duration & 1.315 & 9 & 190 & 0.231 \\
\hline
\end{tabular}

Table 5. Results of one-way ANOVA.

\begin{tabular}{ccccccc}
\hline \multicolumn{2}{c}{ Fixation duration } & Sum of squares & df & Mean square & F & Significance \\
\hline \multirow{2}{*}{$\begin{array}{c}\text { Between } \\
\text { groups }\end{array}$} & Linear comparison & 14628415.127 & 1 & 14628415.127 & 1.863 & 0.174 \\
& term $\quad$ deviation & 150256474.478 & 8 & 18782059.310 & 2.391 & 0.018 \\
& Within group & 1492291446.150 & 190 & 7854165.506 & & \\
& Total & 1657176335.755 & 199 & & & \\
\hline
\end{tabular}

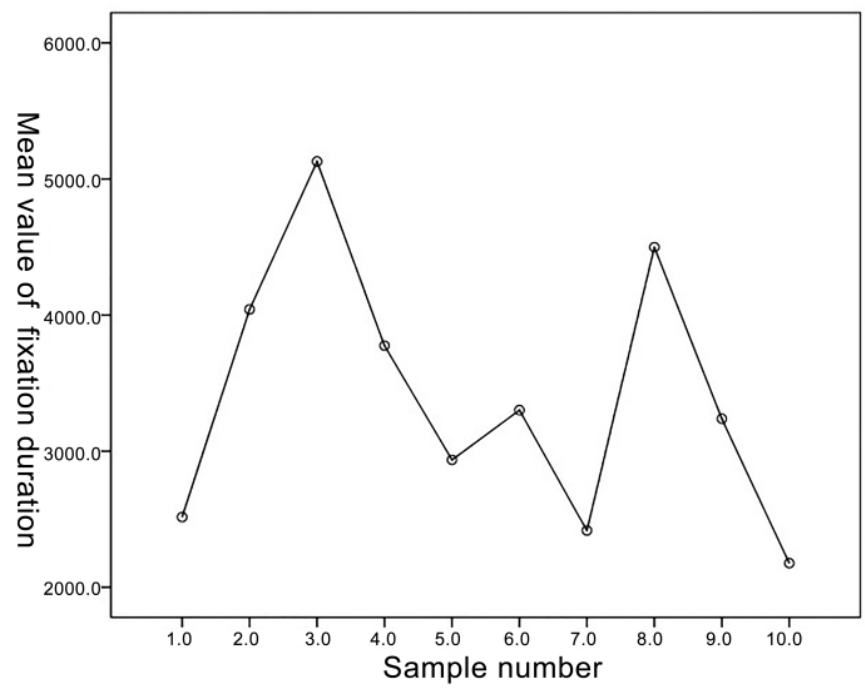

Figure 7. Mean single-element fixation duration results for "solemn—dynamic".

The visual fixation duration data for the remaining six pairs of perception-related words were analyzed as presented in Table 6; the results of one-way ANOVA (p $<0.05)$ revealed that the duration of fixation on the sample images associated with each perception-related word target significantly differed between subjects [18], and that the semantic evaluation data could be subsequently used to establish a neural network model.

Table 6. Results of rectangle difference analysis of perception-related words.

\begin{tabular}{ccc}
\hline & $\mathrm{F}$ & $\mathrm{P}$ \\
\hline solemn - dynamic & 5.562 & 0 \\
exaggerated - simple & 8.524 & 0.036 \\
thin - sturdy & 7.635 & 0.019 \\
streamlined - geometric & 5.032 & 0.002 \\
traditional - modern & 2.333 & 0.016 \\
low-end - high-end & 1.764 & 0.044 \\
\hline
\end{tabular}

\section{Design element decomposition}

According to the Morphological analysis, the design elements of a tractor hood can be implemented as an "item" or a "category" in the perceptual engineering method. After decomposition, eight design elements were obtained: the proportion of the side-plate area, side- plate baseline, top line of the hood, ventilation hole, areal proportion of grille to vehicle cover, side shape of the grille, headlight position relative to the front face of the tractor cover, and headlight shape. The results of the structural decomposition are presented in Table 7. 
Table 7. Decomposition results for hood design elements.

\begin{tabular}{|c|c|c|}
\hline Item & Category & Code \\
\hline \multirow{4}{*}{ A. Proportion of side-plate area } & 1 & 1 \\
\hline & $1 / 2$ & 2 \\
\hline & $2 / 3$ & 3 \\
\hline & $1 / 3$ & 4 \\
\hline \multirow{6}{*}{ B. Side-plate baseline } & Straight line & 1 \\
\hline & Curve & 2 \\
\hline & Fillet plus line & 3 \\
\hline & Curve plus straight line angular transition & 4 \\
\hline & Curve plus curve angular transition & 5 \\
\hline & Straight line plus straight fillet transition & 6 \\
\hline \multirow{5}{*}{ C. Top line of hood } & Fillet plus straight line & 1 \\
\hline & Fillet plus curve & 2 \\
\hline & Edge plus curve & 3 \\
\hline & Forward curve & 4 \\
\hline & Smooth curve & 5 \\
\hline & 2-grid square & 1 \\
\hline \multirow{5}{*}{ D. Ventilation hole } & 3-grid crescent transverse arrangement & 2 \\
\hline & 3-grid square horizontal arrangement & 3 \\
\hline & 3-grid square vertical arrangement & 4 \\
\hline & 4-grid window type & 5 \\
\hline & None & 6 \\
\hline \multirow{5}{*}{$\begin{array}{l}\text { E. Areal proportion of grille to } \\
\text { vehicle cover }\end{array}$} & $1 / 2$ & 1 \\
\hline & $1 / 3$ & 2 \\
\hline & $2 / 3$ & 3 \\
\hline & $3 / 4$ & 4 \\
\hline & 1 & 5 \\
\hline \multirow{5}{*}{ F. Side shape of grille } & Triangle & 1 \\
\hline & Square & 2 \\
\hline & Trapezoid & 3 \\
\hline & Pentagon & 4 \\
\hline & None & 5 \\
\hline \multirow{4}{*}{$\begin{array}{l}\text { G. Headlight position relative to } \\
\text { the front face of the tractor } \\
\text { cover }\end{array}$} & Top & 1 \\
\hline & Middle & 2 \\
\hline & Bottom & 3 \\
\hline & Separate from hood & 4 \\
\hline \multirow{6}{*}{ H. Headlight shape } & Square & 1 \\
\hline & Circular & 2 \\
\hline & Straight line and angular transition & 3 \\
\hline & Straight line and edge curve transition & 4 \\
\hline & Straight line and curve blending fillet transition & 5 \\
\hline & Straight line and curve blending angular transition & 6 \\
\hline
\end{tabular}

The design elements of the 10 tractor hoods were coded according to the decomposition results of the hood design elements presented in Table 7; the results are provided in Table 8 , and they provide data support for the subsequent establishment of an evaluation model for tractor hood designs. 
Table 8. Code list of hood design elements.

\begin{tabular}{ccccccccc}
\hline & A & B & C & D & E & F & G & H \\
\hline Sample 1 & 1 & 3 & 2 & 2 & 2 & 4 & 5 & 5 \\
Sample 2 & 3 & 1 & 1 & 6 & 1 & 5 & 3 & 1 \\
Sample 3 & 2 & 2 & 5 & 6 & 3 & 3 & 1 & 3 \\
$\ldots$ & $\ldots$ & $\ldots$ & $\ldots$ & $\ldots$ & $\ldots$ & $\ldots$ & $\ldots$ & $\ldots$ \\
Sample 8 & 3 & 4 & 3 & 5 & 5 & 3 & 1 & 4 \\
Sample 9 & 2 & 2 & 5 & 6 & 5 & 1 & 4 & 6 \\
Sample 10 & 4 & 4 & 3 & 5 & 5 & 3 & 1 & 4 \\
\hline
\end{tabular}

\section{Network training and prediction}

The BPNN was trained by using the design element category code and the perception-related image evaluation data for the tractor hoods. The eight design element classification codes were implemented as the input layer data, and the average value from the seven representative word group evaluation of each sample image was used as the output layer data. The number of hidden layer nodes was obtained by solving the following empirical formula [19]:

$$
i \leq \sqrt{q \times(t+3)}+1
$$

Where $q$ is the number of input layer nodes; $t$ is the number of output layer nodes; $i$ is the number of hidden layer nodes.

In this study, $p, t$, and $i$ were determined to be 8,7 , and 11 , respectively.

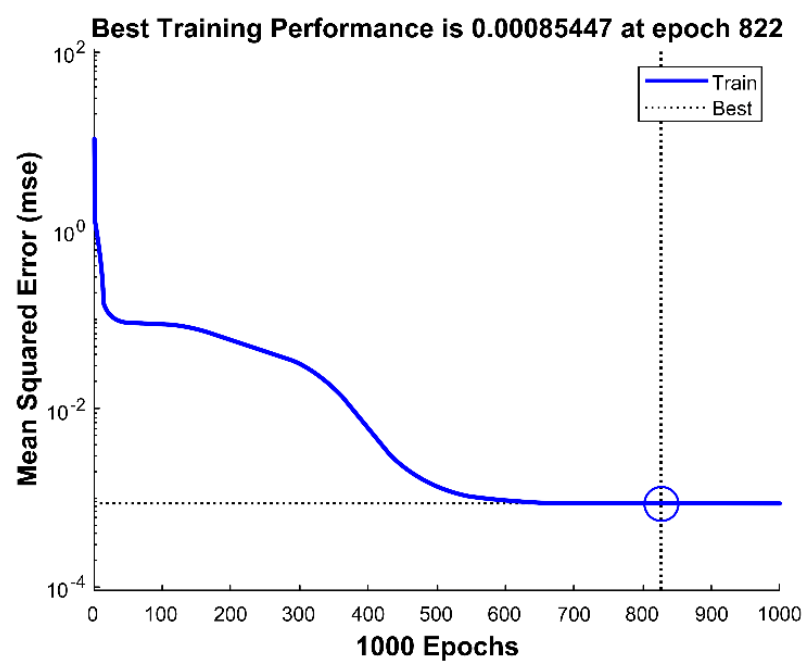

Figure 8. MATLAB network training diagram.

Finally, the trained BPNN was tested on the data for the remaining two sample images; the expected and predicted results are provided in Table 9. Because the perception-based image evaluation was graded using the 7th-order Richter scale, the evaluation axis was divided into six classes, with each score corresponding to 1 point; therefore, the predicted result is acceptable under the condition that its absolute value is less than 0.5. The mean-square error (MSE) of the predictions for the Samples 1 and 2 were 0.1232 and 0.0962 , respectively,
To increase the learning speed and calculation convergence efficiency of the BPNN, the maximum minimum method was employed to normalize the input layer and output layer data [20], as in equation (2):

$$
x_{m}=\left(x_{m}-x_{\min }\right) /\left(x_{\max }-x_{\min }\right)
$$

Where $x_{\min }$ is the minimum, and $x_{\max }$ is the maximum.

Eight sample images were employed as training samples, and the remaining two sample images were employed as test images for network training in MATLAB 2018a. The training results are shown in Figures 8 and 9. Figure 8 shows the MATLAB network training diagram. As shown in the diagram, when the training step reached 822, the error value was 0.00085447 . It can be seen in Figure 9 that the posttraining fit of the network results reached 0.99645, indicating a good fit.

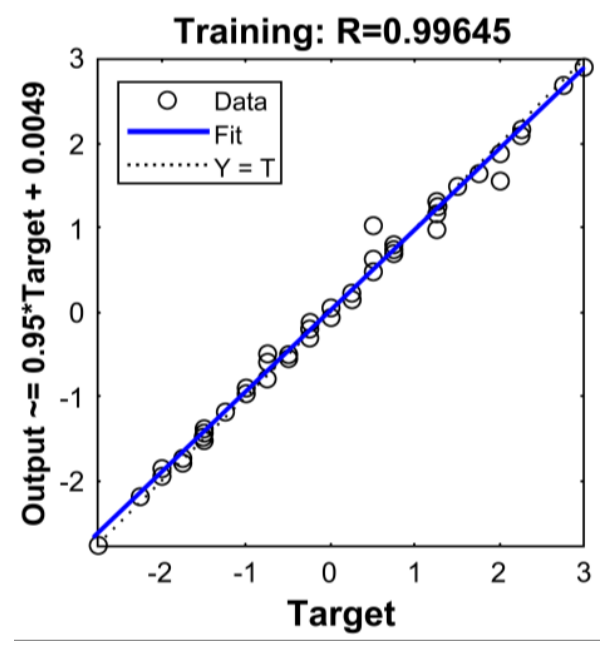

Figure 9. MATLAB network fitness diagram.

indicating that training data on the BPNN yields highly accurate results; this means that the network can be applied for the user evaluation and prediction of tractor hood designs. 
Table 9. Discrepancy between expected and predicted values.

\begin{tabular}{ccccccc}
\hline & \multicolumn{3}{c}{ Sample 1 } & \multicolumn{3}{c}{ Sample 2 } \\
\cline { 2 - 7 }$\cdot$ & actual value & $\begin{array}{c}\text { predicted } \\
\text { value }\end{array}$ & $\begin{array}{c}\text { value of } \\
\text { error }\end{array}$ & actual value & $\begin{array}{c}\text { predicted } \\
\text { value }\end{array}$ & $\begin{array}{c}\text { value of } \\
\text { error }\end{array}$ \\
solemn-dynamic & -1 & -0.5789 & 0.4211 & -1 & -1.3259 & -0.3259 \\
exaggerated-simple & 0.75 & 1.1798 & 0.4298 & 0 & -0.0536 & -0.0536 \\
thin-sturdy & 0.25 & 0.0902 & -0.1598 & 0.5 & 0.3425 & -0.1575 \\
streamlined-geometric & -1.25 & -1.5545 & -0.3045 & 0.25 & -0.1845 & -0.4345 \\
traditional-modern & 0.25 & 0.0165 & -0.2335 & 0.5 & 0.7907 & 0.2907 \\
low-end-high-end & 0 & -0.5082 & -0.5082 & 0.75 & 0.2582 & -0.4918 \\
domestic-international & -0.75 & -0.4874 & 0.2626 & 0.5 & 0.6558 & 0.1558 \\
\hline MSE & \multicolumn{3}{c}{0.1232} & & & 0.0962 \\
\hline
\end{tabular}

\section{Summary}

In this study, eye-tracking technology and BPNNs were used to investigate tractor hood designs. The conclusions are as follows:

(1) By performing eye-tracking experiments, the visual cognition of user observation and evaluation of the tractor shape was obtained, and the most important characteristic of the hood shape was determined.

(2) The task-based eye-movement experiment was carried out with the hood shape as the variable to verify the importance of the hood in user evaluation; additionally, the perception-related image evaluation value was obtained for many subjects via an oral evaluation.

By using structural analysis to decompose the design elements of tractor hoods, and by using user evaluation values and the design element classification code for each hood to train the BPNN, a user evaluation model was established for tractor hoods. The test proves that the model has a high accuracy rate, which can be used to predict the user evaluation of a tractor hood design; it can also be used as a reference for people who design tractor hoods.

\section{References}

1. Guo, H.P. (2018) The form design and kansei evaluation of wheeled tractors based on the front face characteristic. Northwest A\&F University.

2. Liu, Y.K. (2010) Tractor Formative Design and Research on Formative Design Method. Henan University of science and technology.

3. Meng, H., Ruan, Z.H., Liu, Q. (2016) Development history of tractor hood. Tractor \& Farm Transporter, 43: 10-11.

4. Cheng, S.W., Sun, L.Y. (2014) A Survey on Visualization for Eye Tracking Data. Journal of Computer-Aided Design and Computer Graphics, 26: 698-707.

5. Hyeonggyu, P., Sangil, L., Minho, L., Mun-Seon, C., Ho-Wan, K. (2016) Using eye movement data to infer human behavioral intentions. Computers in Human Behavior,63: 796-804.

6. Han, Y.C. (2000) The development of eye tracker and eye movement experiment. Psychological science, 04: 454-457.

7. Guo, G., Lin, L., Xu, N. (2018) User-perceived styling experience of smart vehicles: A method to combine eye tracking with semantic differences. IET Intelligent Transport Systems, 13: 72-78.

8. Qiu, K. (2018) Research on Optimal Design of Product Image Modeling Based on Eye Movement Signal. Lanzhou University of Technology.

9. Li, J., Guo, S.J. (2019) Research on image modeling design and user emotional cognition of humanoid robot. Journal of machine design,36: 134-138.

10. Lu, G.Y., Hou, G.H. (2018) Eye Tracking of Product Design Decision Making Strategy. Packaging engineering, 39: 215-220.

11. Su, J.N., Qiu, K., Zhang, S.T., Xiao L.W., Zhang, X.X. (2017) Evaluation method study of product modeling design elements based on eye movement data. Journal of machine design, 34: 124-128.

12. Zhou, X. (2017) Research on Design Elements of Industrial Robot Based on Kansei Image. Anhui University of Technology.

13. Zhu, Y., Chen, G. (2016) Head shape design of service robots on Kansei engineering and BP neural network. Packaging engineering, 37: 63-67.

14. Li, Y.F., Zhu, L.P. (2019) Research on Product Image Form Design Based on Neural Network. Packaging engineering, 30: 88-90.

15. Su, J.N., Fan, Y.F., Zhang, S.T., OuYang, L.Z. (2011) Product form design based on kansei engineering and neural network. Journal of Lanzhou University of technology, 37: 47-50.

16. Chen, S.B., Yang, S.J., Zhu, L., Li, M.M., Zhang, W.S. (2019) Design and Quantification of Cars Modeling Imagery Based on BP Neural Network. Packaging engineering, 40: 128-133.

17. Xv, J., Sun, S.Q., Zhang, K.J. (2017) Optimization Design of Product Image Model Based on Genetic 
Algorithm. Journal of Mechanical Engineering, 04: 53-64.

18. Xiao, W.Q., Cheng, J.X., Zhou, X., Wang, X.J. (2017) Research on perceptual image space of industrial robot modeling design based on eye movement experiment. Journal of mechine design,34: 124-128.

19. Zhao, W.Q. (2019) Evaluation of product model design based on BP neural network. Computer Engineering and Design,30: 5715-5721.

20. Lu, G.Y., Hou, G.H. (2017) Predictive model of user experience of engineering vehicle modeling design based on eye tracking. Journal of mechanical design, 34: 107-111. 